to be arid formalists and unbridled theorybuilders. She and her daughter tell us that Noam Chomsky, Steven Pinker and hordes of other innatist, structure-oriented investigators have got it all wrong: "This type of reasoning imposes the theory onto the data, rather than letting the data constrain the theory, as good science would dictate." One might retort that few scientific revolutions would have been possible if physicists, chemists and biologists had followed this austere and stultifying precept. And we would surely not have had the so-called cognitive revolution, which was largely spurred on by daring theoretical leaps into the ultimate nature of language, the human mind and the modular organization of natural intelligence. The same can indeed be said for the discovery of innate components of the language faculty that starkly separate a child's linguistic capabilities from general intelligence, social conventions and the practical uses of language.

The authors neither totally reject nor endorse these new ideas; they just keep preaching prudence, prudence, prudence. Several passages advise researchers in the field to proceed with the utmost caution in theorizing about children and language acquisition. As a result, we have a book that appears condescending and uniquely optimized for the training of graduate students intending to work in this field - provided they agree with the authors' ultra-cautious approach.

Massimo Piattelli-Palmarini is in the Department of Linguistics, University of Arizona, Tucson, Arizona 85721, USA.

\section{The birth of matter}

\section{The Magic Furnace: The Search for the Origins of Atoms by Marcus Chown}

Oxford University Press: 2001. 240 pp. \$25

\section{Hans A. Bethe}

The Magic Furnace tells the story of the discovery of the birth of atoms inside stars. Is it a good book? When I was about halfway through, I was doubtful. But the second half, which discusses the build-up of atomic nuclei, is very good. Marcus Chown is happiest when recounting the personal stories of scientists and their achievements. The account of Gustav Kirchhoff's discovery that each element has a characteristic spectrum, and his identification of the elements in the spectrum of sunlight, makes enjoyable reading. And the book reaches its climax with the work of Fred Hoyle and Willy Fowler, who discovered that some atoms were formed in the Big Bang

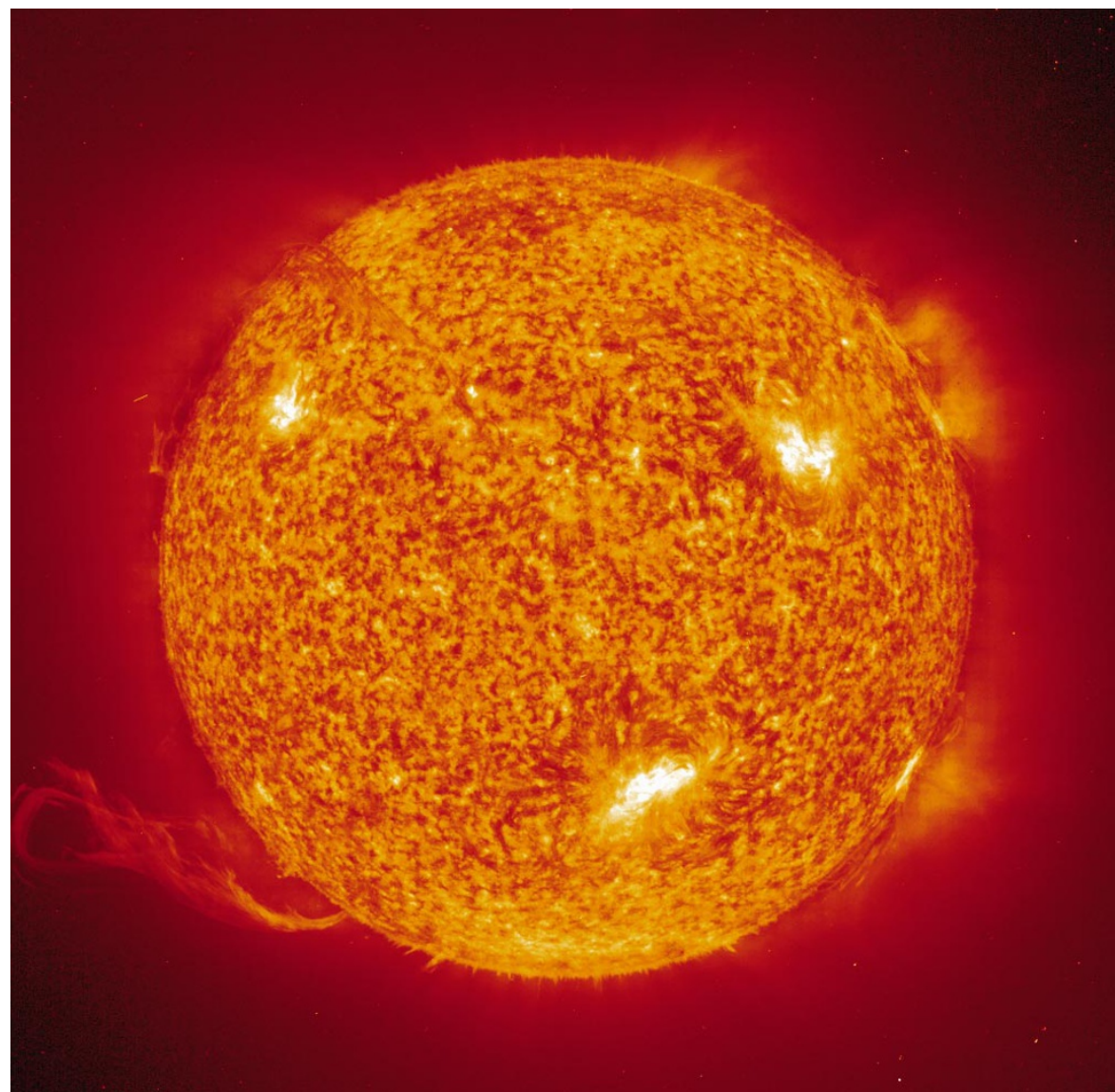

Fiery origins: atoms continue to be forged in the hot interiors of stars.

and others continue to be formed in stars.

The trouble with the first half of the book is that the author does not distinguish clearly enough between new and important findings and those that are well known. Thus, in discussing Arthur Eddington's work on stars, Chown writes the "gas is to generate a high pressure"; it has been known since Robert Boyle's time (1662) that gas has a pressure proportional to its density and temperature. Eddington would not have started his work on stars had he not known this.

Chown describes the carbon-nitrogen cycle and the proton-proton chain, in which hydrogen is converted to helium. But although he tells us that the carbon-nitrogen cycle is sensitive to temperature, we are not told that the temperature at the centre of a star (which can be as much as 40 million degrees kelvin) depends on the material that makes up its bulk. Only much later does the author tell us that stellar material is not like the material on Earth, but is predominantly hydrogen; this reduces the computed central temperature of the Sun from 40 to 13 million degrees kelvin.

The Magic Furnace ends with an informative account of how atoms are made in the extremely hot material in the Big Bang, and in the less hot interiors of stars. It explains why the Big Bang made only helium, and why there is about $25 \%$ (by weight) of helium in the Universe. We discover why there is an abundance of atoms up to the atomic weight of iron, but fewer of those of higher atomic weight, and how the latter are formed in stars by the addition of neutrons to existing nuclei, one by one. And finally we are told how the atoms created in stars escape into the interstellar medium to form new stars.

Hans A. Bethe is emeritus professor of physics,

Cornell University, Ithaca, New York

14853-5001, USA.

\section{Geometry down the centuries}

\section{Euclid's Window: The Story of Geometry from Parallel Lines to Hyperspace}

by Leonard Mlodinow

Free Press: 2001.306 pp. $\$ 26$

\section{Jeremy Gray}

As its subtitle suggests, Euclid's Window aims to take the general reader from elementary ideas of euclidean geometry to Einstein's theory of general relativity and beyond, in this case to the theory of superstrings and their generalizations. Leonard Mlodinow takes a chronological route, describing the mathematics as he goes. So we read a bit 
about euclidean geometry, cartesian coordinate geometry, non-euclidean geometry and special and general relativity in a way that minimizes the technicalities. In the final sections of the book, Mlodinow's own background in physics enables him to introduce some of those involved, such as John Schwarz, Brian Greene and Ed Witten.

Very few proofs or arguments are given. Whether the topic be mathematics or physics, all are presented as descriptions. This leaves the mathematics floating rather more freely than mathematicians might like, because mathematicians are used to explanations and arguments. But a style often found in popular accounts of modern physics may well reach a larger audience, and the interested reader can find the proofs in other books. The information given here is sufficiently accurate, although the account of the Michelson-Morley experiment (which showed that the speed of light does not depend on the direction of transmission) is rather too imprecise. And there are aspects of the book that will not satisfy everyone.

The deliberately light treatment given to the mathematics makes it is almost impossible to connect up the topics in a satisfactory way. Real connections do exist, but Mlodinow has judged them too hard to describe, and the results may not hang convincingly together. The book's determinedly jokey tone becomes less than attractive after a time, and it comes unstuck when attempting to treat the historical material. There are many historical passages, sometimes taken from better recent accounts. But Mlodinow has opted for the storyteller's omnipotent eye: we are at Gauss's bedside as he dies, we are privy to people's thoughts and offered gossip (Schrödinger as the Don Juan of modern physics). Inevitably, there are errors along with insights. But the relentlessly flippant tone undermines the seriousness of the subject. "Iced by the Snow Queen" is not the richest way to describe Descartes' death from pneumonia at the court of Queen Christina of Sweden. And there are many such touches that rob the account of the complexity required to be adequate historically.

Euclid's Window will convince any reader that important material has come out of the centuries of geometry. But readers of Nature may want to start with something more substantial, such as Brian Greene's The Elegant Universe (Vintage, 2000), which starts with Einstein and is a popular book with much more to say.

Jeremy Gray is at the Open University, Milton Keynes MK7 6AA, UK.

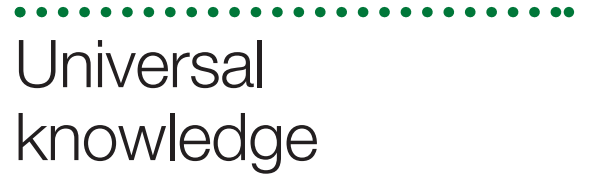

Encyclopedia of Astronomy and Astrophysics, Vols 1-4 editor-in-chief Paul Murdin

Institute of Physics \& Nature Publishing Group: 2001. 3,670 pp. £450, \$650 (printed

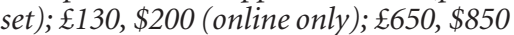
(bundle)

\section{Theodore P. Snow}

Astronomers are fond of referring to their field as the "science of everything", and often boast of their own generality and, well, universal knowledge. Imagine, then, the scope of an encyclopaedia of astronomy and astrophysics - an encyclopaedia of everything. The creation of such a compendium is the task taken on by the publishers of the
Encyclopedia of Astronomy and Astrophysics $(E A A)$. The result is, predictably, massive but successful, at least for a specialized audience.

The EAA is available in print, in four large volumes, and, for subscribers, on the Internet (http://www.ency-astro.com; nonsubscribers can access special features as well as the opening lines of any entry in the encyclopaedia - but they must become subscribers in order to access the full articles). The goals and intended uses of the EAA are set out in Paul Murdin's introduction. The work is aimed at "an exceptionally broad audience" - professionals and students in astronomy, professionals in related fields (such as biology or meteorology), and non-specialists seeking specific information about definitions or biographical information.

Topics are generally treated at quite a high and fairly technical level, in keeping with the aim of providing a useful resource for students and professionals. The content is a mixture of encyclopaedia, dictionary and biographical listing. Short entries defining terms or giving potted biographies are written by staff editors, and unsigned; longer articles on specialized topics are authored by leading researchers. Accuracy is ensured by the expertise of the contributors, who form an impressive who's who of modern astronomy and astrophysics.

The encyclopaedia's coverage is broad and thorough, and in some cases the depth is astonishing. In many areas, multiple entries by different contributors present different facets of the same topic. These create a complete picture that is extremely valuable, even to a specialist. There are no fewer than eight entries by different authors on 'Chromospheres', for example, each devoted to a

\section{New in paperback}

\section{Defenders of the Truth: The Sociobiology Debate \\ by Ullica Segerstråle

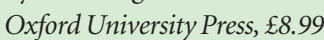 \\ "Segerstråle's goal was to understand the factors that influenced the course of this scientific movement [sociobiology]. On what basis do the sociobiologists as well as their opponents evaluate sociobiology?” David Hull (Nature 407, 673-674; 2000)}

\section{Consciousness: How Matter Becomes} Imagination - previously called A Universe of Consciousness: How Matter Becomes Imagination by Gerald M. Edelman \& Giulio Tononi Penguin, $£ 8.99$

"Explaining consciousness has become the Holy Grail of modern neuroscience. Any reckoning on who has found the true path is surely premature. Nevertheless, the account of consciousness provided by Edelman and Tononi is certainly highly plausible and can be recommended as one of the most ambitious accounts around." Raymond J. Dolan (Nature 407, 450-451; 2000)

The Voice of Genius: Conversations with Nobel Scientists and Other Luminaries

by Denis Brian

Perseus, $£ 14.99$

\section{Stepping Stones: The Making of Our Home World}

by Stephen Drury

Oxford University Press, $£ 12.99$

Charles Darwin's Beagle Diary

edited by R. D. Keynes
Cambridge University Press, $£ 14.95, \$ 21.95$

\author{
Darwin's Ghost: The Origin of \\ Species \\ by Steve Jones \\ Ballantine, \$15
}

Lucy's Legacy: Sex and Intelligence in Human Evolution

by Alison Jolly

Harvard University Press, \$18.95, £12.95

The Sun, the Genome and the Internet by Freeman J. Dyson

Oxford University Press, $£ 6.99, \$ 10.95$

Bold Science: Seven Scientists Who Are Changing Our World

by Fred Anton

W. H. Freeman, $\$ 14.95$ 